\title{
Stripline design for the extraction kicker of Compact Linear Collider damping rings
}

\author{
C. Belver-Aguilar and A. Faus-Golfe \\ Instituto de Física Corpuscular, 46980 Paterna, Spain \\ F. Toral \\ Centro de Investigaciones Energéticas, Medioambientales y Tecnológicas, 28040 Madrid, Spain \\ M. J. Barnes \\ European Organization for Nuclar Research CERN, Geneva 23, CH-1211 Switzerland
}

(Received 3 December 2013; published 31 July 2014)

\begin{abstract}
In the framework of the design study of future linear colliders, the Compact Linear Collider (CLIC) aims for electron-positron collisions with high luminosity at a nominal center-of-mass energy of $3 \mathrm{TeV}$. To achieve the luminosity requirements, predamping rings (PDRs) and damping rings (DRs) are required: they reduce the beam emittance before the beam is accelerated in the main linac. Several kicker systems are needed to inject and extract the beam from the PDRs and DRs. In order to achieve both low beam coupling impedance and reasonable broadband impedance matching to the electrical circuit, striplines have been chosen for the kicker elements. In this paper, we present the complete design of the striplines for the DR extraction kicker, since it is the most challenging from the field homogeneity point of view. The excellent field homogeneity required, as well as a good transmission of the high voltage pulse through the electrodes, has been achieved by choosing a novel electrode shape. With this new geometry, it has been possible to benefit from all the advantages that the most common shapes introduce separately. Furthermore, a detailed study of the different operating modes of a stripline kicker allowed the beam coupling impedance to be reduced at low frequencies: this cannot be achieved by tapering the electrodes. The optimum design of the striplines and their components has been based on studies of impedance matching, field homogeneity, power transmission, beam coupling impedance, and manufacturing tolerances. Finally, new ideas for further improvement of the performance of future striplines are reported.
\end{abstract}

DOI: 10.1103/PhysRevSTAB.17.071003

PACS numbers: 41.85.Ar, 41.75.Ht

\section{INTRODUCTION}

High-energy electron-positron colliders, such as CLIC, will be needed to investigate the $\mathrm{TeV}$ physics revealed by the LHC. To achieve high luminosity at the interaction point (IP), it is essential that the beams have very low vertical emittance: predamping rings (PDRs) and damping rings (DRs) will damp the beam emittance to extremely low values, via the process of radiation damping.

In CLIC, a total of four rings, two PDRs and two DRs, will be required to damp both the electron beam and the positron beam. Both PDRs and DRs have a racetrack configuration. The length of each ring is 389.15 and $427.5 \mathrm{~m}$ for the PDRs and DRs, respectively [1].

The PDRs are needed to accommodate the large emittance bunches from the electron/positron injectors, and damp them to emittances that are small enough to be

Published by the American Physical Society under the terms of the Creative Commons Attribution 3.0 License. Further distribution of this work must maintain attribution to the author(s) and the published article's title, journal citation, and DOI. injected into the DR without losses. For electrons, the PDRs will damp the normalized beam emittance from 100 to $63 \mu \mathrm{m}$, in the horizontal plane, and from 100 to $1.5 \mu \mathrm{m}$, in the vertical plane. For positron beams, the PDRs are more challenging: they have to damp the input emittance of $7 \mathrm{~mm}$ to the same output emittances as for the electrons, in both vertical and horizontal planes. The role of the DRs is to provide the final stage of damping to the required low emittance at a fast repetition rate of $50 \mathrm{~Hz}$. The normalized emittance requirements are extremely low, $500 \mathrm{~nm}$ in the horizontal plane and $5 \mathrm{~nm}$ in the vertical plane, in spite of the high bunch intensity of $4.1 \times 10^{9}$. Once the beam is damped to the final emittance values, it is extracted from the DRs and injected into the booster linac, where the beam will be accelerated from 2.86 to $9 \mathrm{GeV}$ [1].

Most of the parameter choices of the CLIC DRs are driven by the requirement for the unprecedented low emittances. The main design parameters of the PDRs and DRs, for electrons, are summarized in Table I.

The injection and extraction process will be carried out using one injection and one extraction system in each ring, with only one pulse stored in the rings per cycle. This pulse 
TABLE I. Beam parameters and kicker specifications for the PDRs and DRs for electrons [1].

\begin{tabular}{|c|c|c|c|}
\hline Beam parameters & Symbol & $\begin{array}{l}\text { PDRs } \\
1 / 2 \mathrm{GHz}\end{array}$ & $\begin{array}{c}\text { DRs } \\
1 / 2 \mathrm{GHz}\end{array}$ \\
\hline Beam energy (GeV) & $E$ & 2.86 & 2.86 \\
\hline Circumference (m) & $C$ & 389.15 & 427.5 \\
\hline Bunch population $\left(10^{9}\right)$ & $N_{b}$ & 4.3 & 4.1 \\
\hline \multirow[t]{2}{*}{ Normalized emittance $(\mu \mathrm{m})$} & $\gamma \epsilon$ & $63(\mathrm{H})$ & $0.5(\mathrm{H})$ \\
\hline & & $1.5(\mathrm{~V})$ & $0.005(\mathrm{~V})$ \\
\hline Bunches per train & & $156 / 312$ & $156 / 312$ \\
\hline Bunch spacing (ns) & & $1 / 0.5$ & $1 / 0.5$ \\
\hline Bunch length (mm) & $\sigma_{b}$ & $3.2 / 4.6$ & 1.6/1.8 \\
\hline Kicker parameters & Symbol & $\begin{array}{l}\text { PDRs } \\
1 / 2 \mathrm{GHz}\end{array}$ & $\begin{array}{c}\text { DRs } \\
1 / 2 \mathrm{GHz} \\
\end{array}$ \\
\hline Deflection angle (mrad) & $\alpha$ & 2.0 & 1.5 \\
\hline Aperture (mm) & $a$ & 40 & 20 \\
\hline Effective length (m) & $L$ & 3.4 & 1.7 \\
\hline Field rise and fall time (ns) & & $428 / 1000$ & $560 / 1000$ \\
\hline Pulse flat top (ns) & & $900 / 160$ & $900 / 160$ \\
\hline Flat top reproducibility & & $\pm 1 \times 10^{-4}$ & $\pm 1 \times 10^{-4}$ \\
\hline Injection stability & & $\pm 2 \times 10^{-2}$ & $\pm 2 \times 10^{-3}$ \\
\hline Extraction stability & & $\pm 2 \times 10^{-3}$ & $\pm 2 \times 10^{-4}$ \\
\hline $\begin{array}{l}\text { Injection } \\
\quad \text { inhomogeneity (\%) }\end{array}$ & & $\pm 0.1^{\mathrm{a}}$ & $\pm 0.1^{\mathrm{a}}$ \\
\hline $\begin{array}{l}\text { Extraction } \\
\quad \text { inhomogeneity }(\%)\end{array}$ & & $\pm 0.1^{\mathrm{a}}$ & $\pm 0.01^{\mathrm{b}}$ \\
\hline Repetition rate $(\mathrm{Hz})$ & $f_{\text {rep }}$ & 50 & 50 \\
\hline Vacuum (mbar) & & $10^{-10}$ & $10^{-10}$ \\
\hline Stripline voltage (kV) & $V_{k}$ & \pm 17.0 & \pm 12.5 \\
\hline Stripline current (A) & $I_{k}$ & \pm 340 & \pm 250 \\
\hline $\begin{array}{l}\text { Longitudinal beam } \\
\text { impedance }(\Omega / n)\end{array}$ & $Z_{\|}$ & 0.05 & 0.05 \\
\hline $\begin{array}{l}\text { Trans. beam imped. } \\
\qquad(\mathrm{k} \Omega / \mathrm{m})\end{array}$ & $Z_{\perp}$ & 200 & 200 \\
\hline
\end{tabular}

${ }^{\mathrm{a} O v e r} 3.5 \mathrm{~mm}$ radius.

${ }^{\mathrm{b}}$ Over $1 \mathrm{~mm}$ radius.

contains either two trains of 156 bunches with $1 \mathrm{GHz}$ rf structure, or one single train of 312 bunches with $2 \mathrm{GHz}$ rf structure. The injection and extraction system consist of a FODO (a magnet structure consisting of focusing and defocusing quadrupoles with drift spaces between the quadrupoles) cell with kicker and septum magnets in the drift spaces to deflect the beam. The injection and extraction kickers are located at symmetric locations, after the dispersion suppressor and upstream of the superconducting wigglers, to avoid damage from synchrotron radiation [2].

In this paper, we will discuss in detail the extraction kicker from the CLIC DRs. This kicker will be the first one to be prototyped because the kick field uniformity required is at least one order of magnitude better than in the other cases, although for a smaller region, as is shown in Table I. Once a first prototype of the extraction kicker from the DRs is built and validated, it should be feasible to prototype the striplines for the other PDR and DR kickers with only small variations in the design. The choice of the kicker parameters and the selection of a stripline-type kicker is explained in Sec. II. Taking into account these parameters, and the electromagnetic response of a pair of electrodes, shown in Sec. III, the design of the kicker is reported in Sec. IV. Furthermore, studies of the materials and components for the kicker fabrication are discussed in Sec. V. Finally, in Sec. VI, studies towards improvement of future striplines are presented.

\section{KICKERS PARAMETER CHOICE AND TECHNOLOGY}

In the DRs, the quadrupole magnets have a circular aperture of $20 \mathrm{~mm}$ diameter, and the dipoles and wigglers have an elliptical aperture with vertical gaps of 20 and $13 \mathrm{~mm}$, respectively [1]. To minimize the impact of the DR extraction kicker on the overall machine beam coupling impedance, the same beam pipe cross section is used as for the downstream quadrupoles. For that reason, a circular aperture of $20 \mathrm{~mm}$ diameter was selected for the aperture of the striplines of the CLIC DRs extraction kicker.

Any ripple or droop on the extraction kicker pulse will produce beam size jitter which could be propagated up to the IP of the collider. The extraction kicker has a tolerance for the beam jitter of less than $10 \%$ of the beam size, hence the relative deflection stability requirement can be calculated as $\delta \theta / \theta=0.1 \sigma_{x} / x_{\text {sep }}$, where $\sigma_{x}$ is the beam size at the extraction and $x_{\text {sep }}$ is the position of the beam with respect to the center of the septum, which is approximately $9 \mathrm{~mm}$. Taking into account that the extracted beam size for the required normalized emittance of $500 \mathrm{~nm}$ corresponds to a few tens of $\mu \mathrm{m}$, the kicker stability is of the order of $10^{-4}$ [1]. This kicker stability refers to the extraction uniformity and flat top reproducibility reported in Table I.

The complete beam pulse will be extracted at the same time. Therefore, the flat top corresponds to the length of the bunch train for the $2 \mathrm{GHz}$ baseline, or the length of the two bunch trains plus the train separation for the $1 \mathrm{GHz}$ baseline. The CLIC DRs beam coupling impedance requirements make the stripline technology an ideal solution for extracting the CLIC DRs high current beams. The allowable broad band impedances in the CLIC PDRs and DRs are $1 \Omega / n$, where $n$ is the number of turns, for longitudinal beam coupling impedance, and $10 \mathrm{M} \Omega / \mathrm{m}$ in the transverse plane. These requirements will guarantee the beam stability against single bunch effects [3]. Since the allowable beam impedance is for a complete DR, which is composed of many systems including both injection and extraction kicker systems, the permissible beam coupling impedances, per kicker system, are assumed to be $5 \%$ of the longitudinal impedance allowance, i.e. $0.05 \Omega / n$, and $2 \%$ of the transverse impedance allowance, i.e. $200 \mathrm{k} \Omega / \mathrm{m}$ [4]. These specifications for the beam coupling impedance make the stripline technology an ideal solution for the kicker systems of the CLIC PDRs and DRs. 


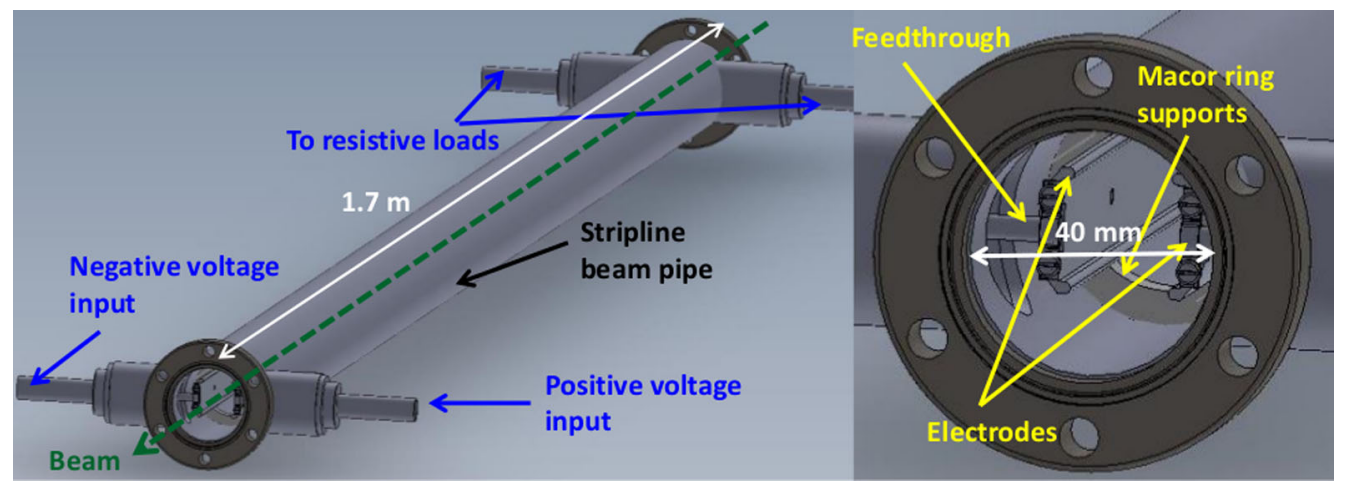

FIG. 1. 3D model of a stripline kicker.

The deflection angle $\alpha$ and the beam energy $E$ determine the transverse voltage required to deflect the beam: $V_{\perp}=E \tan \alpha$. Supposing that the electric field $\mathcal{E}$ is constant along the striplines, the required value can be calculated using the transverse voltage $V_{\perp}$ and the stripline length $L: \mathcal{E}=V_{\perp} / 2 L$. From the electric field the required voltage $V_{k}$ required to be applied to each electrode can be estimated, since the electrodes are pulsed to equal magnitude but opposite polarity voltage: $V_{k}=\mathcal{E} \times a / 2$, where $a$ is the aperture of the striplines.

The characteristic impedance of the striplines has been chosen to be $Z_{0}=50 \Omega$, since it represents an optimum compromise between power transmission and energy losses [5]. In addition, this characteristic impedance is a standard value and thus commercial components are available. The striplines will be driven by an inductive adder: a first prototype has been built and is being tested at CERN [6]. Ideally the characteristic impedance of the inductive adder, the feedthroughs, the striplines, and the terminating resistors must be matched to avoid reflections, which could cause ripple on the flat top of the deflection pulse. For an electrode terminated in $Z_{0}$ the current is directly related to the electrode voltage by means of the characteristic impedance: $I_{k}=V_{k} / Z_{0}$.

\section{STRIPLINE KICKER OPERATION}

The striplines proposed for the extraction kicker of the CLIC DRs consists of two parallel electrodes housed in a conducting cylinder: each of the electrodes is driven by an equal but opposite polarity pulse, produced by an inductive adder [6]. The striplines will be powered, via coaxial feedthroughs, from the beam exit end: the upstream feedthroughs will be connected to resistive loads. A 3D model of the stripline kicker prototype is shown in Fig. 1.

Each of the stripline electrodes with its adjacent ground planes (beam pipe walls) forms a transmission line for transverse electromagnetic waves. Therefore, the stripline kicker operates as two coupled transmission lines, each of which should ideally have a characteristic impedance matched to $50 \Omega$. Coupled transmission line theory shows two operating modes for the stripline kicker, since three conductors are involved in the signal transmission, i.e. both electrodes and the vacuum beam pipe: these modes are known as odd and even modes. When the electrodes are excited with equal magnitude but opposite polarity voltages, the current flow is in opposite directions in each stripline electrode and an electromagnetic field is created between the electrodes, giving a transverse kick to the beam: this is the odd mode (Fig. 2, top). When an unkicked circulating beam passes through the aperture of the striplines, it induces image currents in the electrodes: the direction of current flow is the same in both electrodesthis is the even mode (Fig. 2, bottom). The induced current generates an electromagnetic field, which gives a longitudinal kick to the beam and can produce beam instabilities.

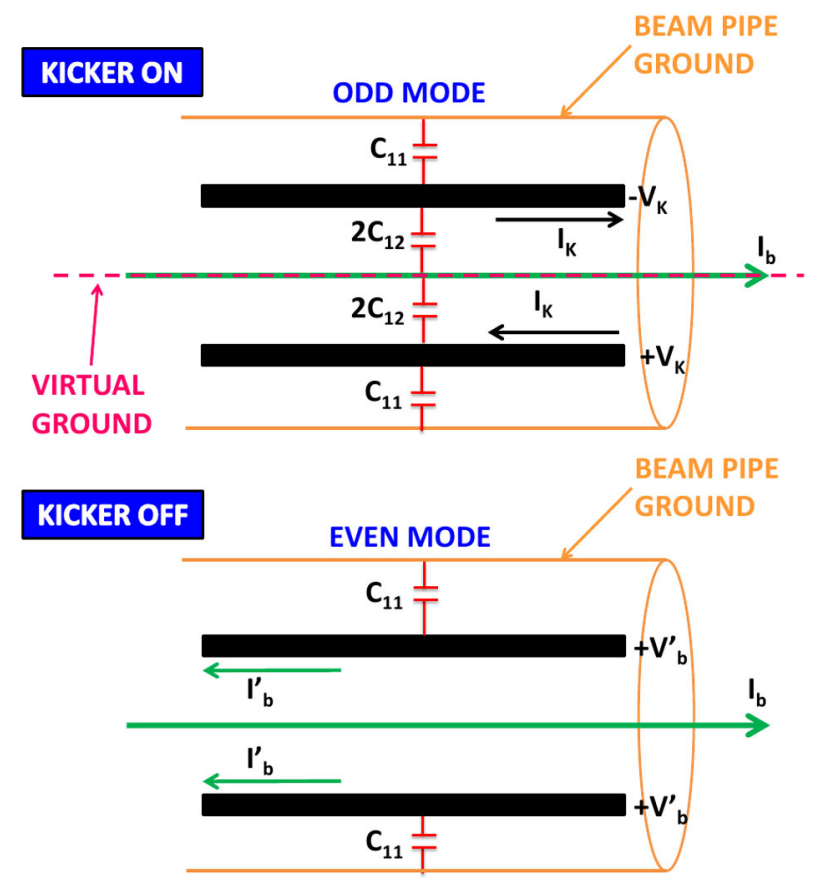

FIG. 2. Scheme of the two operation modes of a pair of coupled electrodes: odd mode (top) and even mode (bottom). For the even mode, $I_{b}^{\prime}$ and $V_{b}^{\prime}$ corresponds to the image current and voltage created by the beam when passing through the aperture of the striplines. 


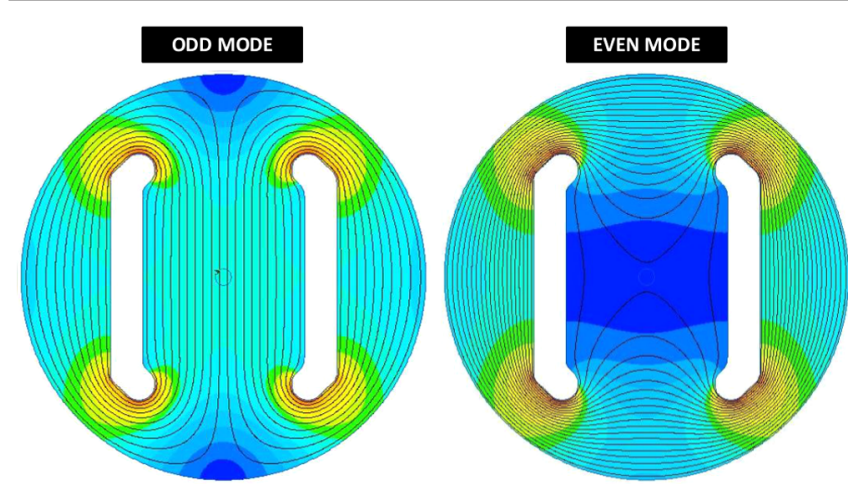

FIG. 3. Electric field pattern for the odd (left) and even (right) modes. The color pattern goes from orange (maximum electric field) to dark blue (zero electric field).

Each mode has its own electromagnetic field pattern and its own characteristic impedance: the field pattern associated with these modes is shown in Fig. 3.

Both operation modes must be taken into account when optimizing the characteristic impedance of the striplines. For the odd mode, the electric field lines have an odd symmetry about the center line, and a voltage null exists between the two electrodes (Fig. 3). Hence, there is a virtual ground plane midway between the electrodes: the capacitance between an electrode and the virtual ground is $2 C_{12}$ (Fig. 2, top). In this case, the effective capacitance between an electrode and ground is $C_{\text {odd }}=C_{11}+2 C_{12}$, and the characteristic impedance is $Z_{\text {odd }}=1 /\left(c C_{\text {odd }}\right)$, where $c$ is the speed of light. In the even mode, since the two electrodes are at the same potential, there is no charge stored in the capacitance between them. The resulting capacitance of either electrode to ground for the even mode is $C_{\text {even }}=C_{11}$ (Fig. 2, bottom). Therefore, the characteristic impedance for the even mode is $Z_{\text {even }}=1 /\left(c C_{\text {even }}\right)$.

\section{DESIGN OF THE STRIPLINE GEOMETRY}

The extraction kicker system of the CLIC DRs demands very tight stability tolerances in order to extract the low emittance beam, in a very stable and reproducible way. When starting the striplines design study, and due to this tight stability tolerance, we identified several challenges that made it impossible to reuse designs previously reported in the literature. The most important challenges were the following: (i) the excellent field homogeneity required, with only $\pm 0.01 \%$ of inhomogeneity allowed over $1 \mathrm{~mm}$ radius, in the center of the aperture; (ii) good power transmission, with extremely low ripple, by achieving good impedance matching of the striplines in the deflecting (odd) mode; (iii) a very low beam coupling impedance, which should be below $0.05 \Omega / n$ in the longitudinal plane and $200 \mathrm{k} \Omega / \mathrm{m}$ in the transverse plane: the beam coupling impedance is important during the nondeflecting (even) mode.

Injection and extraction stripline kickers have already been used for fast injection/extraction applications, such as bunch-by-bunch manipulation, with a field rise time of a few ns. This is the case of the tapered striplines designed for the injection upgrade of the $\Phi$ factory DA $\Phi N E$ [7], as well as the kicker system proposed for the ILC DR beam extraction [8]: both designs allow for field rise times below 6 ns. For the CLIC DR extraction kicker, the curved and tapered electrodes used in DA $\Phi$ NE would give higher field inhomogeneity than is permissible. In addition, tapering has been demonstrated to increase the beam coupling impedance at very low frequencies, and this is undesirable for CLIC. On the other hand, due to the high energy of the beam in the CLIC DRs, deflecting the beam by the specified $1.5 \mathrm{mrad}$ requires a long stripline kicker.

As explained in Sec. II, the CLIC DR extraction will not be bunch-by-bunch, but a complete train of 156 (or 312) bunches will be extracted at the same time; hence the required rise time for the CLIC DR kickers is 2 orders of magnitude larger than the DA $\Phi$ NE injection kicker and the ILC DR extraction kicker. However, low beam coupling impedance requirements make the stripline technology an ideal solution for injecting/extracting high current beams. This is also the case for the CTF3 combiner ring (CR) extraction kicker [9]. The geometry of the CTF3 CR extraction kicker, which has the same stripline length but larger aperture ( $39 \mathrm{~mm}$ instead of $20 \mathrm{~mm}$ ), has been studied for the CLIC DR extraction kicker, though not all the specifications were met, as shown in Sec. IV E.

To increase the field homogeneity over a wide aperture, an electrostatic injection kicker has been developed and installed in the KEK digital accelerator [10]. However, an electrostatic kicker is not the most suitable solution for the CLIC DRs extraction kicker, as it makes use of only the electric field, whereas a stripline kicker makes use of both electric and magnetic fields to deflect the beam, making stripline kickers more efficient than electrostatic kickers.

In [7-10] only the deflecting (odd) mode has been optimized to have $50 \Omega$ characteristic impedance, therefore obtaining a good power transmission. Optimizing the nondeflecting (even) mode of the striplines will reduce the beam coupling impedance. A design for good matching of both the odd and even mode characteristic impedances is being developed for the Advanced Photon Source Upgrade (APS-U) injection kicker, [11]: good matching is achieved by introducing ground fenders in the cross section of the striplines. These ground fenders are not useful in our case: with these it is not possible to achieve the excellent field homogeneity required for the extraction kicker of the CLIC DRs. Furthermore, due to the small aperture, fenders would be very close to the electrode edges, so the electric field in those regions would be too high. In addition, due to the long striplines, ground fenders should be manufactured as an independent part, and it would be difficult to assemble them with high accuracy.

Hence we took a different approach to optimizing the striplines, and studied the dependence of the field 
homogeneity, as well as the odd and even mode characteristic impedances, upon the cross section geometry of the striplines. This approach, using the capacitances $\mathrm{C}_{11}$ and $\mathrm{C}_{12}$ defined in Sec. III, has turned out to be a simple, but very effective, method to understand how the odd/even characteristic impedances change with the electrode shapes. This study, explained in Sec. IVA, has allowed us to achieve the excellent field homogeneity, good matching of both characteristic impedances, and a decrease in the beam coupling impedance at low frequencies, by designing a new electrode shape. All these studies have been carried out by using three different simulation codes: High Frequency Structure Simulator (HFSS) [12], Computer Simulation Technology (CST) [13]—both Particle Studio (PS) and Microwave Studio (MS), and QuickField [14].

\section{A. Characteristic impedance and field homogeneity optimization}

The geometric cross section of the striplines defines the characteristic impedance and the homogeneity of the electromagnetic field in the aperture.

The characteristic impedance of both odd and even modes should ideally be optimized to $50 \Omega$. However for coupled electrodes this is not possible to achieve. The difference between the even mode and odd mode characteristic impedances is due to the capacitance between the electrodes $\left(C_{12}\right)$, and an infinite separation between the electrodes would be needed to uncouple the electrodes, i.e. $C_{12}=0$. Since the beam coupling impedance is directly proportional to the even mode characteristic impedance, as is shown in Sec. IV D, it is desirable that the even mode impedance is not larger than $50 \Omega$. Hence a goal is to minimize the ratio $C_{12} / C_{11}$ in order to diminish the difference between the even and odd mode characteristic impedances. Ensuring that the odd mode characteristic impedance of each electrode is close to $50 \Omega$ will avoid large mismatches to the characteristic impedance of feedthroughs, coaxial cables, and the inductive adder. This will help to minimize reflections when pulses are applied to energize the electrodes (Sec. IV C).

The electrode cross section was selected by studying several shapes for the striplines and optimizing each shape to achieve $50 \Omega$ even mode characteristic impedance, and $\pm 0.01 \%$ field inhomogeneity over a circle of $1 \mathrm{~mm}$ radius at the center of the aperture. The odd mode impedance of each optimized shape was also calculated.

The most common electrode shapes for striplines, used in kickers, are flat and curved electrodes [7-11]. Flat electrodes show good field homogeneity, whereas curved electrodes may allow for a better impedance matching between the two operation modes, but with poorer field homogeneity. Hence a new geometry was proposed: the half-moon electrode. This new electrode shape allows for both features, i.e. good field homogeneity and suitable

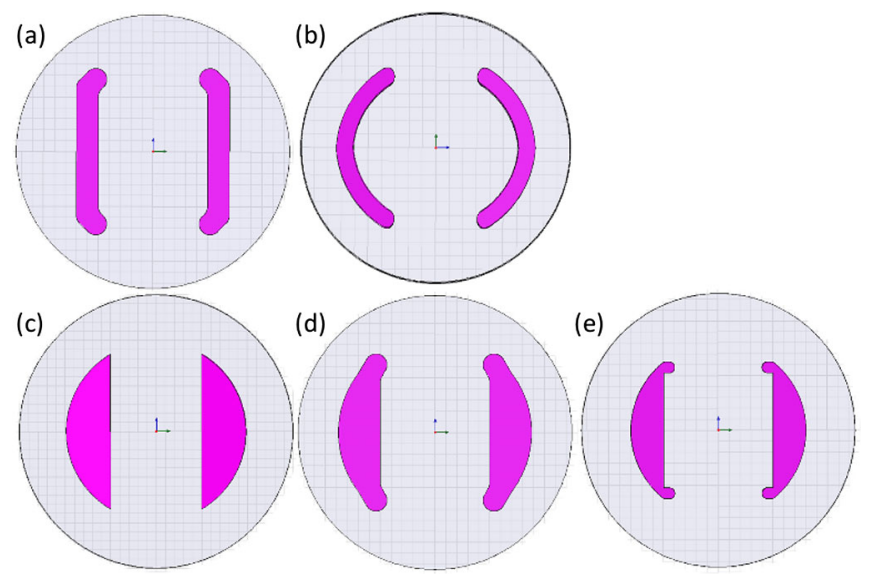

FIG. 4. Electrode shapes studied: flat electrodes (a), curved electrodes (b), and half-moon electrodes (c)-(e).

impedance matching. The five electrode cross sections considered for the design of the striplines are shown in Fig. 4: all of them have a cylindrical beam pipe, since it is the easiest shape for manufacturing from a commercial pipe.

Computer simulations have been carried out by changing the ratio between the electrode height ( $h$ in Fig. 5) and the stripline beam pipe inner radius $R$, for three different pipe radii: 20,25 , and $30 \mathrm{~mm}$. The minimum beam pipe radius considered is $20 \mathrm{~mm}$ since this is the minimum to avoid potential problems of electric breakdown between the electrodes and beam pipe: the acceptable minimum distance between electrode and beam pipe has been determined based on the Kilpatrick limit [15], though this criterion is usually pessimistic.

For a first-order analytical approximation the electrodes can be considered as parallel plate capacitors, where edge effects and fringe fields are neglected; in addition, the capacitance between an electrode and the beam pipe can be thought of as the capacitance between a strip conductor and an infinite ground plane. Therefore, for calculating $C_{12}$, the capacitance of a parallel plate $C=\epsilon_{0} A / a$ has been used, where $\epsilon_{0}=8.854 \times 10^{-12} \mathrm{~F} / \mathrm{m}$ is the permittivity of free space, $A$ is the surface area of one plate, and $a$ is the distance between plates, i.e. the aperture. For calculating $C_{11}$, i.e. the capacitance between an electrode and an infinite ground plane, Eq. (1) [16] has been used:

$$
Z_{\text {even }}=\frac{1}{c C_{11}}=\left(\frac{87}{\sqrt{\epsilon_{r}+1.41}}\right) \ln \left(\frac{5.98 d}{0.8 h+w}\right),
$$

where $\epsilon_{r}=1$ is the relative permittivity of the vacuum, $d$ is the distance between the electrode and the beam pipe wall (value calculated from the center of the electrode height $h$ ), and $w$ is the electrode thickness. All these parameters are shown in Fig. 5.

The analytical results, shown in Fig. 6, are as expected: it is confirmed that a small stripline beam pipe radius results in closer values of even and odd mode characteristic 

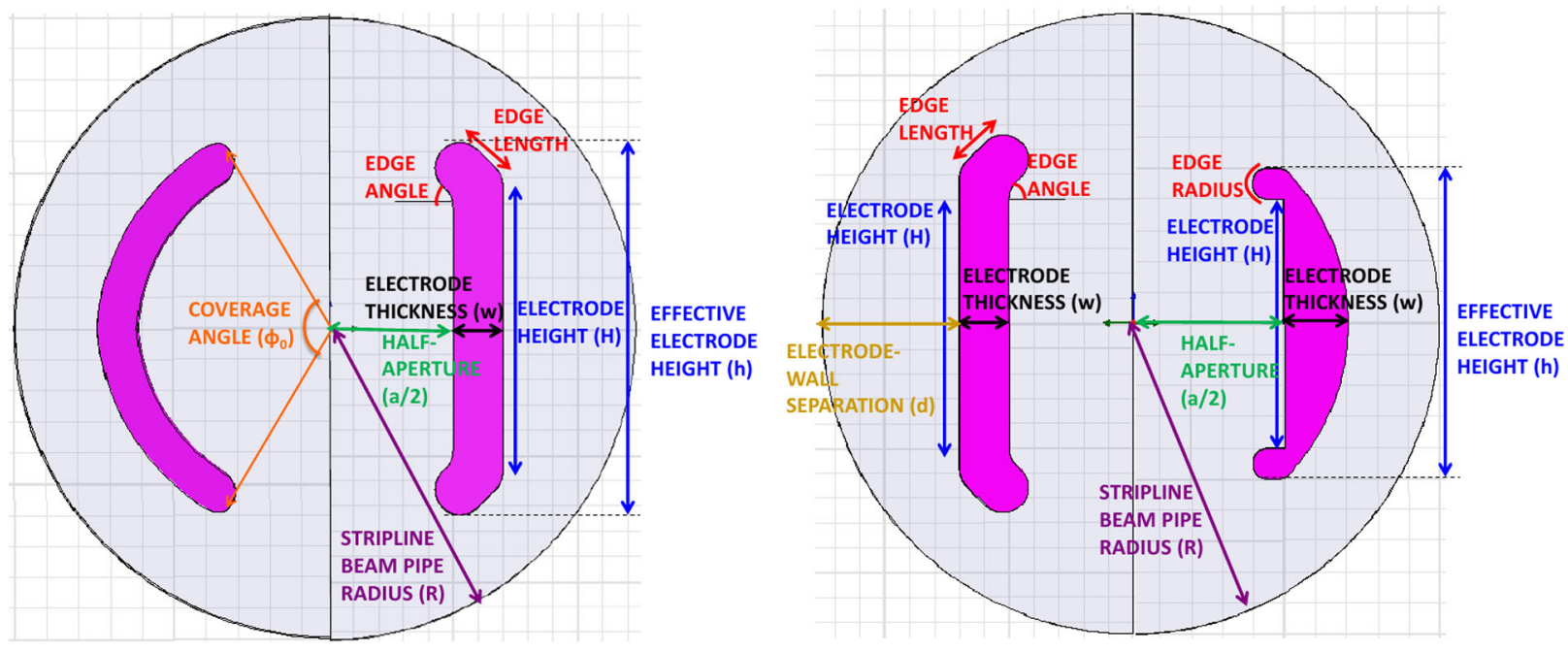

FIG. 5. Geometric parameters used to optimize the shape of the electrodes: the schematic on the left shows the parameters for a curved and a flat electrode, and the schematic on the right for a flat and a half-moon electrode type (e).

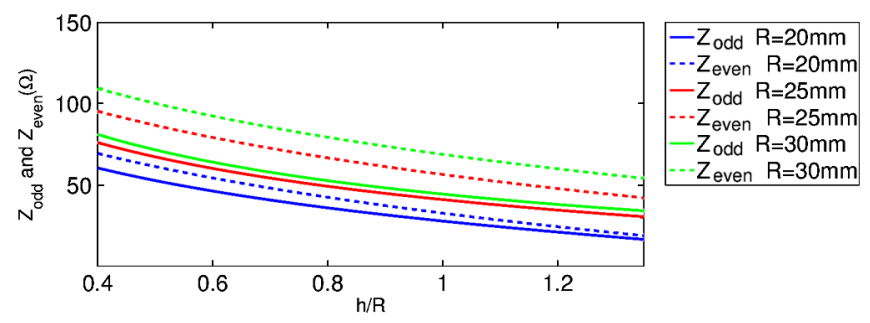

FIG. 6. Analytical estimate for the odd and even modes characteristic impedance versus $h / R$.

impedance; in addition, the even mode characteristic impedance of striplines is always higher than the odd mode.

For flat electrodes [Fig. 4(a)] and type (e) half-moon electrodes [Fig. 4(e)], the characteristic impedance and field homogeneity have been simulated with HFSS (a finite element method solver for electromagnetic structures) [12]. The results are shown in Figs. 7 and 8, respectively. The main results are collected in Table II. For curved electrodes, the equivalent parameter to $h / R$ is the coverage angle, defined in Fig. 5.

The results for the characteristic impedance, shown in Fig. 7, are as expected: it is confirmed that a small stripline beam pipe radius results in closer values of even and odd mode characteristic impedance; in addition, the even mode

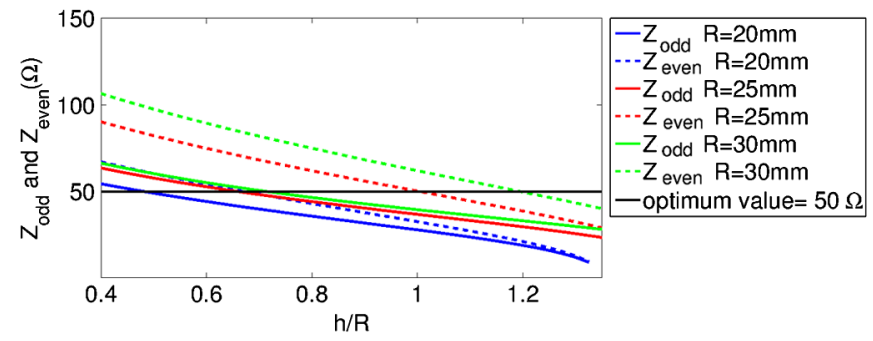

characteristic impedance of striplines is always higher than the odd mode.

Figure 7 shows a similar behavior of the characteristic impedance to that calculated from the analytical approximation (Fig. 6), especially for the flat electrode. For halfmoon electrodes, the odd mode impedances are all equal (Fig. 7, right).

As shown in Table II, with flat electrodes and both type (d) and type (e) half-moon electrodes (Fig. 4) it is possible to achieve the required field homogeneity. For flat electrodes with $50 \Omega$ even mode characteristic impedance, an odd mode characteristic impedance of $36.8 \Omega$ was achieved with a stripline beam pipe radius of $25 \mathrm{~mm}$. For the type (d) and (e) half-moon electrodes the odd mode characteristic impedance is 34.2 and $40.9 \Omega$, respectively. The higher odd mode impedance for the type (e) half-moon electrodes is due to the fact that the distance between the electrode and the stripline beam pipe, optimized for field homogeneity, is smaller than for either the flat electrodes or the type (d) half-moon electrodes. Hence, the ratio $C_{11} / C_{12}$ is higher for the type (e) half-moon electrodes, which leads to closer values for the odd mode and even mode characteristic impedances.

Therefore, flat and type (e) half-moon electrodes (from now on, half-moon electrodes) have been studied further and, in the following sections of the paper, the features of

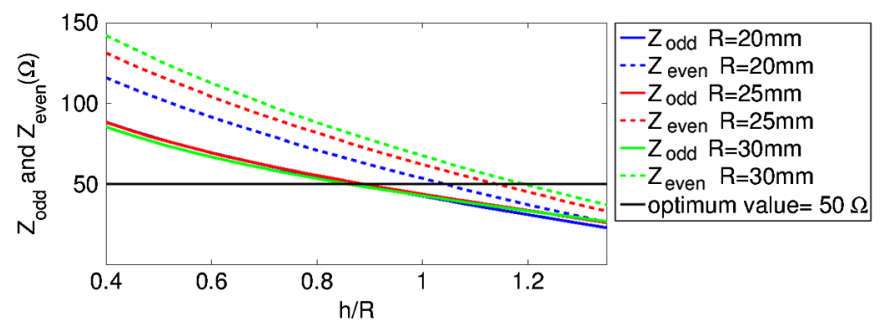

FIG. 7. Odd and even mode characteristic impedance versus h/R, for flat electrodes (left) and type (e) half-moon electrodes (right). 

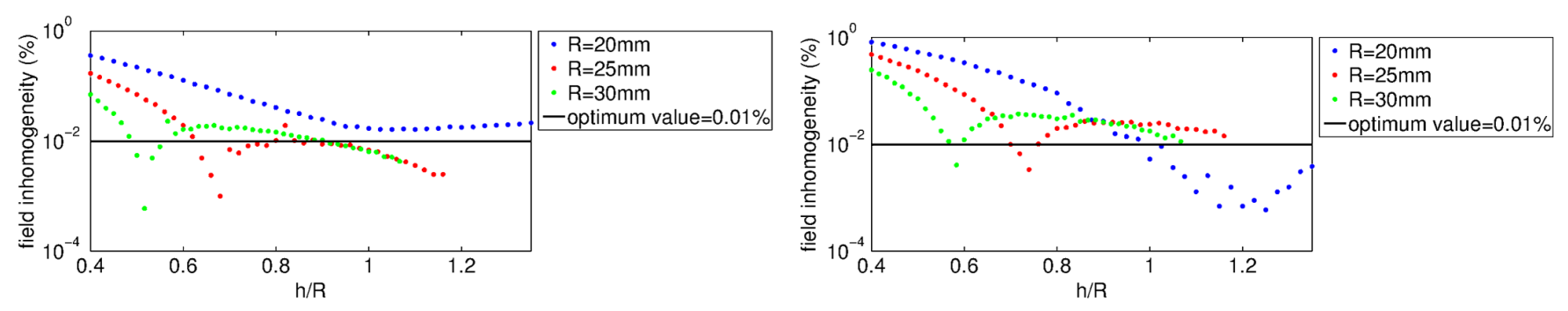

FIG. 8. Odd mode field inhomogeneity (logarithmic scale) over $1 \mathrm{~mm}$ radius versus $\mathrm{h} / \mathrm{R}$, for flat electrodes (left) and type (e) halfmoon electrodes (right). For 25 and $30 \mathrm{~mm}$ radius, field homogeneity simulations produced invalid results for values of h/R $\geq 1.1$.

both electrode shapes are compared, which allows the final geometric design of the striplines to be chosen.

\section{B. Power transmission through the striplines}

A total of four coaxial feedthroughs are required to transfer power from the inductive adders to the two electrodes and from the electrodes to the two $50 \Omega$ loads (Fig. 1).

The feedthroughs are coaxial outside of the beam pipe but the connection from a feedthrough to an electrode cannot be coaxial: hence the characteristic impedance of the connection to the electrode is not $50 \Omega$. Furthermore, during kicker operation, the electrode (odd mode) characteristic impedance is lower than $50 \Omega$. These impedance mismatches result in reflections: the impedance of the connection to the electrode can be calculated using $S$ parameters. HFSS has been used to calculate the reflection parameter $S_{11}$, looking into an input port when the corresponding output port is resistively terminated with $50 \Omega$. The results of the simulations are shown in Fig. 9. The peaks of the reflection parameter increase with frequency, with more impact for the flat electrode. The shape of the curves depends on the coaxial feedthrough to electrode transition, and the frequency difference between peaks is related to the electrode length as $\Delta f=c / 2 L \approx 90 \mathrm{MHz}$. The half-moon electrode has a reflection magnitude below 0.35 over the whole frequency range analyzed (up to $1 \mathrm{GHz}$ ), whereas the flat electrode shape has a reflection parameter consistently below 0.35 only up to approximately $350 \mathrm{MHz}$.

\section{Settling time}

In this paper the inductive adder is considered to generate trapezoidal pulses of rise time $T_{r}$. As mentioned above,

TABLE II. Optimum values for the geometric parameters of the striplines studied, for a $Z_{\text {even }}=50 \Omega$.

\begin{tabular}{lcccc}
\hline \hline Configuration & $\begin{array}{c}\mathrm{R} \\
(\mathrm{mm})\end{array}$ & $\mathrm{h} / \mathrm{R}$ & $\begin{array}{c}Z_{\text {odd }} \\
(\Omega)\end{array}$ & $\begin{array}{c}\text { Field inhomogeneity } \\
(\%)\end{array}$ \\
\hline Flat (a) & 25 & 1.0 & 36.8 & $< \pm 0.01$ \\
Curved (b) & 20 & 2.6 & 37.0 & \pm 0.65 \\
Half-moon (c) & 30 & 0.6 & 35.2 & \pm 0.015 \\
Half-moon (d) & 30 & 1.0 & 34.2 & $< \pm 0.01$ \\
Half-moon (e) & 20 & 1.05 & 40.9 & $< \pm 0.01$ \\
\hline \hline
\end{tabular}

impedance mismatches create reflections and thus ripple. The time for the ripple to reduce within specification $( \pm 0.02 \%)$ is called the settling time: in this paper settling time is measured from the end of the rise time $(0 \%$ to $100 \%$ ) of the idealized trapezoidal current pulse considered to be produced by the inductive adder.

From the inductive adder point of view, settling time should be as low as possible; settling time increases the required pulse width, thus increasing power dissipation and the cross-sectional area of magnetic material. Hence the aim is to limit settling time $\left(\mathrm{T}_{s}\right)$ such that it is no more than $100 \mathrm{~ns}$.

Figure 10 shows the predicted settling time versus odd mode characteristic impedance of the electrodes, for $0 \%$ to $100 \%$ rise times of the output current pulse of the inductive adder of 50, 100, and $150 \mathrm{~ns}$ : for these simulations the output pulse is modeled as being trapezoidal. Furthermore, the inductive adder and coaxial cable to striplines have a total single way delay of $13 \mathrm{~ns}$, whereas the striplines have a single way delay of $7 \mathrm{~ns}$. The faster the rise time of the output pulse is, from the inductive adder, in general, the longer the settling time is. For flat electrodes, an odd mode characteristic impedance of $36.8 \Omega$ results in a settling time of $113 \mathrm{~ns}$ for a rise time of the output pulse, from the inductive adder, of $100 \mathrm{~ns}$. For the same rise time, a settling time of $78 \mathrm{~ns}$ will result when the odd mode characteristic impedance is $40.9 \Omega$, which is the case for the half-moon electrodes. Therefore, half-moon electrodes allow for a reduced settling time, which is beneficial for the design of the inductive adder.

For an ideal trapezoidal waveform there will be high frequency components, due to the discontinuities at the

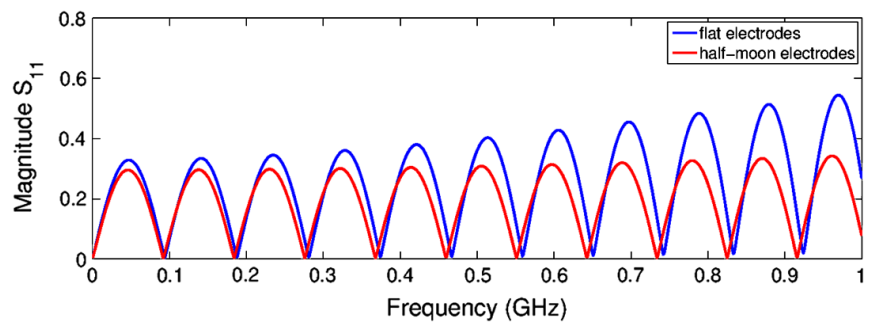

FIG. 9. $S_{11}$ looking into an input port, when the corresponding output is terminated with $50 \Omega$, for flat electrodes (blue) and halfmoon electrodes (red), for a frequency range from dc to $1 \mathrm{GHz}$. 


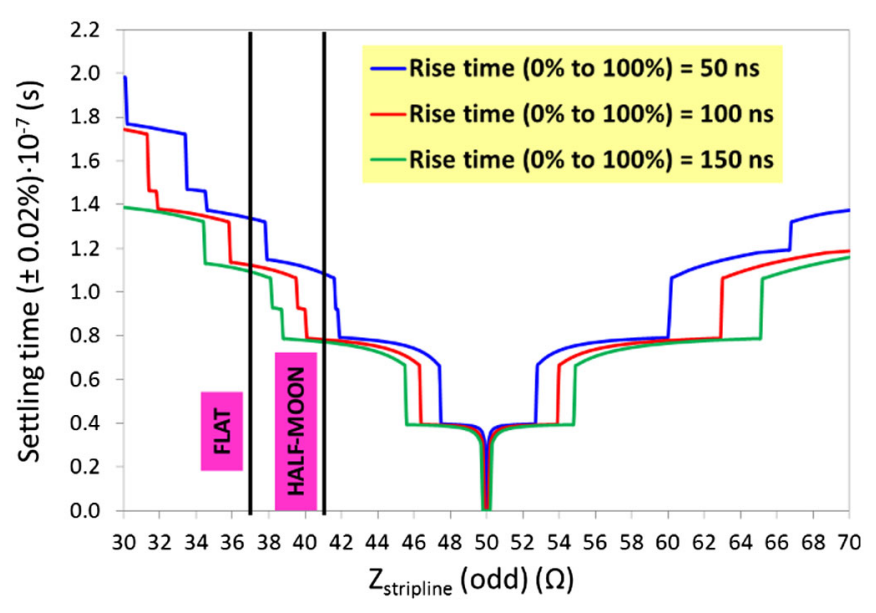

FIG. 10. Settling time of the stripline voltage pulse as a function of odd mode stripline impedance for different $0 \%$ to $100 \%$ rise times of the output pulse of the inductive adder. The inductive adder, transmission line, and terminating resistor impedances are $50 \Omega$.

inflections. In a realistic model these discontinuities do not exist; therefore, the real pulse might have a different time response than the ideal shape which might give different results in Fig. 10. However, the simplified trapezoidal waveform gives a "worst-case" approach to determining an acceptable odd mode impedance for the striplines.

\section{Beam coupling impedance}

A charged particle beam traveling inside a vacuum chamber induces electromagnetic fields, known as wakefields, which act back on the beam itself. This gives an additional voltage and energy gain and hence affects the longitudinal dynamics: in addition there is a transverse momentum kick which deflects the beam.

Beam coupling impedance is a very important parameter for the striplines. Since the DRs are periodic, it is important to reduce as much as possible the effect of the field produced by the beam itself, in the striplines, when the kicker is not operating, in order to diminish the wakefield effects in the unkicked circulating beam. Knowledge of the electromagnetic interactions, between the charged particle beam and the vacuum chamber, is necessary in order to avoid the instability phenomena that may otherwise occur in the CLIC DR.

By using the code CST Particle Studio, the beam coupling impedance has been studied. Results for untapered striplines are shown in Fig. 11.

The results show that the magnitude of the low frequency peak for the longitudinal beam coupling impedance is lower in the case of half-moon electrodes. This is due to the fact that the longitudinal beam coupling impedance is proportional to the square of the coverage angle [17] for each half-moon electrode the coverage angle is $1.8 \mathrm{rad}$ compared to $2 \mathrm{rad}$ for a flat electrode. Transverse beam
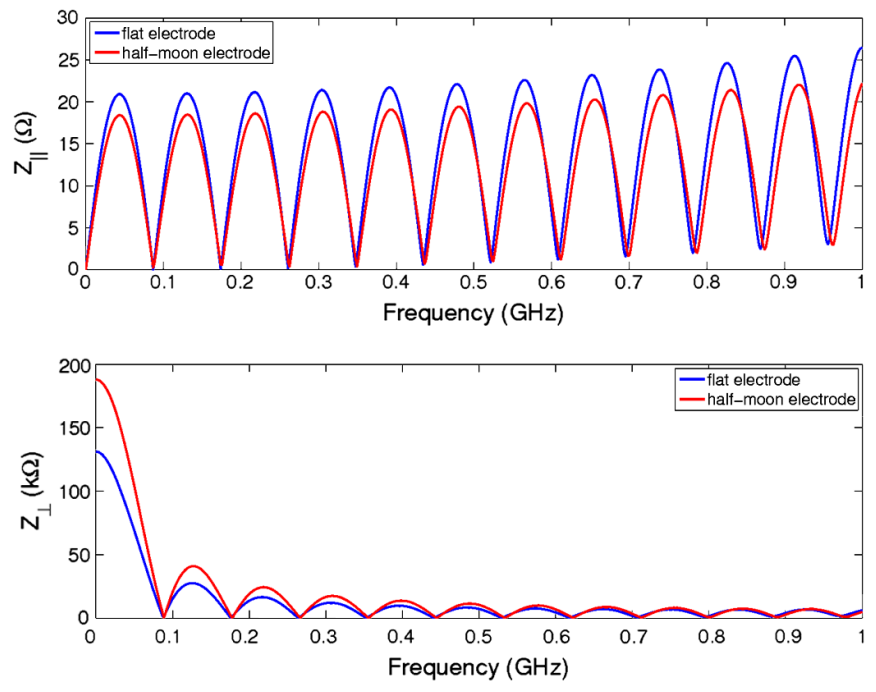

FIG. 11. Longitudinal (top) and transverse (bottom) beam coupling impedance results for untapered striplines from CST Particle Studio simulations for flat electrodes (blue) and halfmoon electrodes (red).

coupling impedance is lower in the case of flat electrodes, since the stripline beam pipe radius is larger in this case [17], as it is shown in Table II.

\section{E. Discussion and choices of the striplines geometry}

Studies of the cross section of the striplines for the extraction kicker of the CLIC DRs have shown that for a $50 \Omega$ even mode characteristic impedance of the striplines and the field homogeneity required, the half-moon electrode shape results in an odd mode characteristic impedance closer to $50 \Omega$ than the flat electrodes (Table II). Furthermore, the reflection coefficient predicted looking into the input port, with $50 \Omega$ on each output port, shows that the transmission is slightly better in the case of halfmoon electrodes (Fig. 9), hence the settling time (Fig. 10) is reduced. Finally, simulations of beam coupling impedance show that the longitudinal beam coupling impedance is lower for the half-moon electrode, whereas the flat electrode shape is better from the transverse beam coupling impedance point of view (Fig. 11).

Overall, the half-moon electrodes are considered as the best choice for the cross section of the striplines for the extraction kicker of the CLIC DRs. The possibility of a next prototype with tapered striplines will be discussed in Sec. VI A.

\section{MANUFACTURING TOLERANCES, MATERIALS, AND COMPONENT STUDIES}

The prototype striplines have been manufactured under the Spanish Program "Science for Industry." The geometry chosen for the prototype striplines consists of the optimized 
half-moon electrodes inside a commercial beam pipe of $20.25 \mathrm{~mm}$ inner radius.

In the following, the manufacturing tolerances, as well as the material and component choices for the prototype stripline kicker are presented.

\section{A. Material choices}

The beam pipe housing the stripline electrodes is a stainless steel tube of $1.712 \mathrm{~m}$ length. The internal diameter of the tube is $40.5 \pm 0.2 \mathrm{~mm}$. Aluminium has been chosen for the electrodes, because it is relatively easy to achieve the tight tolerances required during manufacturing. The electrode supports will be manufactured using Macor, which is a machinable glass ceramic [18]. All the materials and components chosen, such as feedthroughs, are compatible with ultrahigh vacuum, of the order of $10^{-10}$ mbar.

\section{B. Study and optimization of electrode supports}

The electrodes have a total length of $1.639 \mathrm{~m}$ and, ideally, must be perfectly aligned along their entire length. Cylindrical supports have been used in other stripline kickers with larger stripline beam pipe radius, such as the CTF3 extraction kicker where the inner radius of the stripline beam pipe was $39 \mathrm{~mm}$ [9]; however, in the CLIC DR design cylindrical supports will not be used, because it is not possible to achieve the required precision for the electrode alignment. In order to ensure the alignment a new solution has been proposed, which consists of fixing the electrodes outside the aperture by using four equally spaced Macor rings, of $10 \mathrm{~mm}$ length each. Once the electrodes are aligned and fixed to the Macor rings, this assembly will be placed inside the stainless steel tube, and its angular position will be guaranteed by a pin embedded in the stripline beam pipe wall. The assembly of the electrodes outside the vacuum chamber allows the dimensions to be controlled using a 3D machine and hence guarantees the field homogeneity, which is very difficult to measure in the laboratory using electromagnetic methods.

The number of Macor rings and their length has been selected by studying the mechanical requirements and the impedance mismatch introduced by the rings. This impedance mismatch introduced by the Macor rings results in power being reflected: the reflection parameter has been simulated using CST MS and HFSS.

Figure 12 shows that the Macor rings increase the magnitude of the reflection parameter $S_{11}$, starting from $300 \mathrm{MHz}$, of every third peak. The separation between these maxima corresponds to the distance, there and back, between the equally spaced Macor rings $(510 \mathrm{~mm})$. The frequency content of the driving pulse from the inductive adder will only extend up to approximately $f=0.35 / T_{r} \approx$ $7 \mathrm{MHz}$, where the pulse rise time considered is $T_{r}=50 \mathrm{~ns}$. Thus, since the Macor rings mainly affect the $S_{11}$ above $300 \mathrm{MHz}$, they are not expected to significantly influence the ripple of the driving pulse.

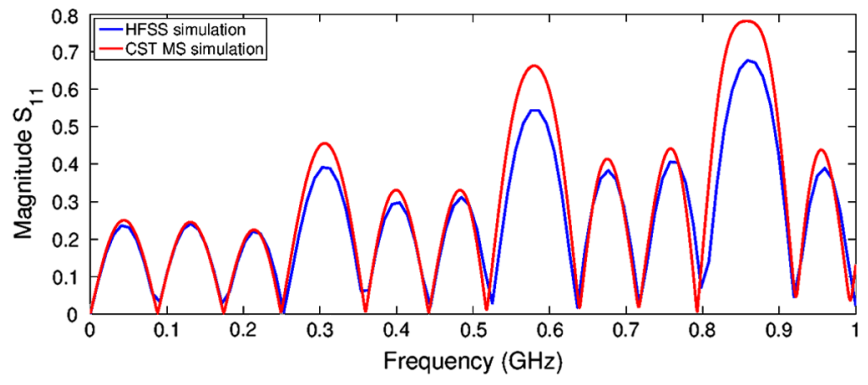

FIG. 12. Magnitude of $S_{11}$ predicted by HFSS and CST MS, for half-moon electrode striplines with four equally spaced Macor ring supports, and ideal $50 \Omega$ feedthroughs.

The presence of the Macor rings will also affect the uniformity of the electric field. HFSS simulations show that the field inhomogeneity increases from $\pm 0.008 \%$ to $\pm 0.07 \%$ in the regions where the Macor is placed. Since the 4 rings are $10 \mathrm{~mm}$ long, the total length occupied by this ceramic is $40 \mathrm{~mm}$, which corresponds to $\approx 2.5 \%$ of the total striplines length. Hence the field inhomogeneity over the $1 \mathrm{~mm}$ radius, integrated along the length of the striplines, is $\pm 0.009 \%$, which is within specification.

From the beam coupling impedance point of view, the presence of the Macor rings increases the energy lost by the beam particles when passing through the aperture. Hence there is an increase of the longitudinal beam coupling impedance above $\approx 330 \mathrm{MHz}$ (Fig. 13, top), whereas the transverse beam coupling impedance (Fig. 13, bottom) is not significantly affected because the Macor rings do not change the cross section of the striplines.
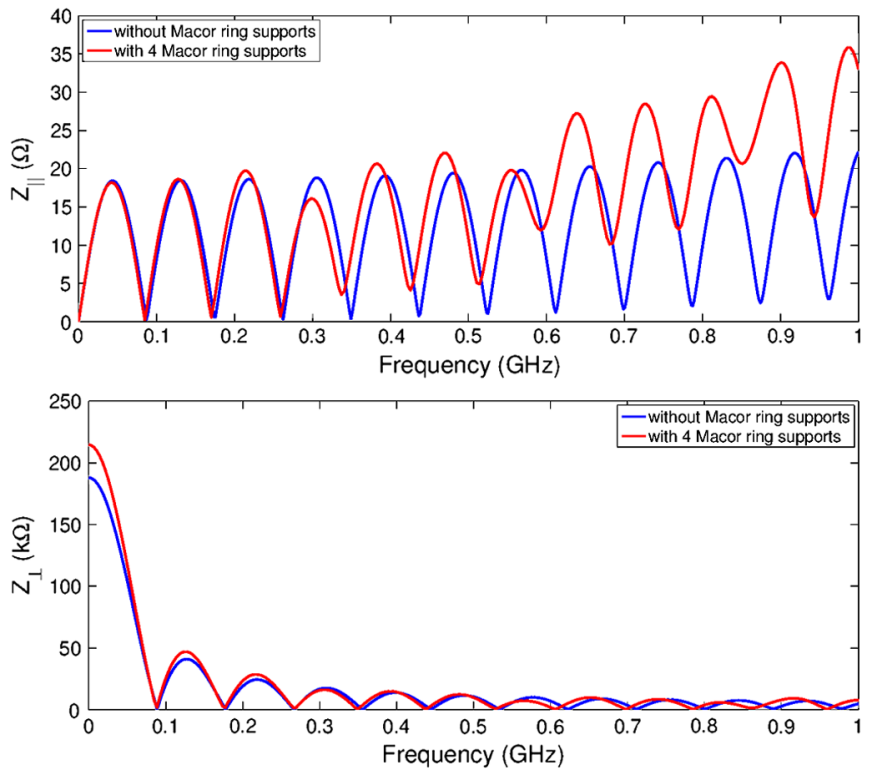

FIG. 13. Longitudinal (top) and transverse (bottom) beam coupling impedance for half-moon electrode striplines with and without Macor rings, simulated with CST PS, for ideal $50 \Omega$ feedthroughs. 


\section{Fabrication tolerances}

The effect of fabrication tolerances, upon field homogeneity over a $1 \mathrm{~mm}$ radius, has been studied using the $2 \mathrm{D}$ code Quickfield. The geometric errors considered are the horizontal and vertical positions and the tilt angle of an electrode. All these parameters are mechanically defined by the tolerances of the electrode supports and the electrodes (see Fig. 14). The horizontal aperture of the Macor rings is $25.50 \mathrm{~mm}$ with a tolerance of $+0 /-0.10 \mathrm{~mm}$, and the electrode thickness is $2.75 \mathrm{~mm}$ and has a tolerance of $+0.05 /-0.05 \mathrm{~mm}$. Hence, a maximum error in the horizontal position of the electrodes of $+0.20 \mathrm{~mm}$ is expected. The vertical position is defined by the diameter of the Macor ring in the vertical dimension, $40.30 \mathrm{~mm}$ with a tolerance of $+0 /-0.10 \mathrm{~mm}$, as well as the thickness of the ring, which is $14.40 \mathrm{~mm}$ with a tolerance of $+0 /+0.05 \mathrm{~mm}$. This leads to a maximum error expected in the vertical position of the electrodes of $0.15 \mathrm{~mm}$. Finally, a nonparallelism of the electrodes will occur if the two flat parts of the Macor ring are not parallel with each other. Each flat part of the ring could be tilted by $0.05 \mathrm{~mm}$, which can result in a nonparallelism of one electrode with respect to the other one. In addition, the flat part of the electrode could also have a nonparallelism of $0.05 \mathrm{~mm}$. Then, a maximum nonparallelism of $0.1 \mathrm{~mm}$ in the horizontal plane is expected. Since the electrode height is $\mathrm{H}=20.6 \mathrm{~mm}$, we have that the maximum tilt angle expected in one electrode is $\tan \theta=0.1 / H$ which corresponds to $0.06^{\circ}$. If the assembly of electrodes is rotated inside the beam stripline radius, it will not affect the field inhomogeneity, but it will change the orientation of the electromagnetic field, producing an error in the deflection direction.

Table III summarizes the field inhomogeneity results for three different types of manufacturing tolerances studied.

The maximum field inhomogeneity specified, for the CLIC extraction DR kicker, over a $1 \mathrm{~mm}$ radius, is $\pm 0.01 \%$. As shown in Table III, a manufacturing error in the horizontal position of an electrode may increase the field inhomogeneity beyond specification, whereas an error in the

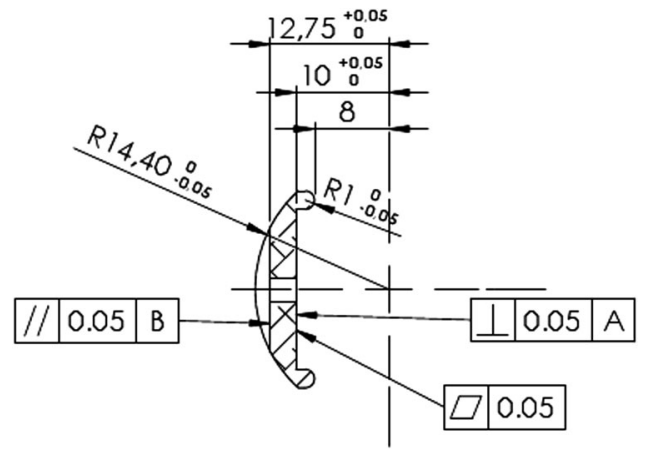

FIG. 14. Fabrication tolerances for the electrodes.
TABLE III. Field inhomogeneity, over a $1 \mathrm{~mm}$ radius, for the maximum geometrical errors expected during manufacturing.

\begin{tabular}{lcc}
\hline \hline & $\begin{array}{c}\text { Maximum } \\
\text { error }\end{array}$ & $\begin{array}{c}\text { Field } \\
\text { inhomogeneity }\end{array}$ \\
\hline None & 0 & $\pm 0.003 \%$ \\
Horizontal (H) & $+0.2 \mathrm{~mm}$ & $\pm 0.012 \%$ \\
Vertical (V) & $-0.15 \mathrm{~mm}$ & $\pm 0.007 \%$ \\
Tilt (T) & $0.06^{\circ}$ & $\pm 0.008 \%$ \\
Combination $(\mathrm{H}+\mathrm{V}+\mathrm{T})$ & & $\pm 0.015 \%$ \\
\hline \hline
\end{tabular}

vertical position of an electrode or a tilt angle of $0.06^{\circ}$ will not. A combination of these three errors, however, would increase the field inhomogeneity to $\pm 0.015 \%$. Therefore, the field inhomogeneity requirement has been studied and relaxed: the kicker good field region has been reduced from $1 \mathrm{~mm}$ radius to $0.5 \mathrm{~mm}$ radius [19]. A study of fabrication tolerances to achieve a field inhomogeneity of $\pm 0.01 \%$ over $0.5 \mathrm{~mm}$ radius has commenced.

\section{Feedthroughs study and optimization}

A total of four feedthroughs will connect the stripline electrodes with an inductive adder and terminating resistors [6]. In the ideal case, in order to maximize power transmission, the impedance of the striplines, feedthroughs, inductive adder, and resistive terminator would be matched to $50 \Omega$ at all frequencies. Furthermore, good impedance matching will reduce the settling time (Fig. 10).

The transition between the feedthroughs and electrodes is important from the good pulse transmission point of view, especially at high frequencies: an abrupt impedance change between the feedthroughs and the electrode can affect the beam coupling impedance. To study the effects of the feedthroughs upon the power reflected, a model with ideal coaxial feedthroughs was first used. Once we chose the Kyocera 15kV-F-UHV feedthrough [20] (Fig. 15), it was simulated (Figs. 16 and 17), and results are shown in Fig. 18.

Figure 18 shows that up to $\approx 800 \mathrm{MHz}$ the magnitude of the $S_{11}$ parameter is generally lower with the commercial feedthroughs than with the ideal feedthroughs. This is due to the impedance of the connection between the

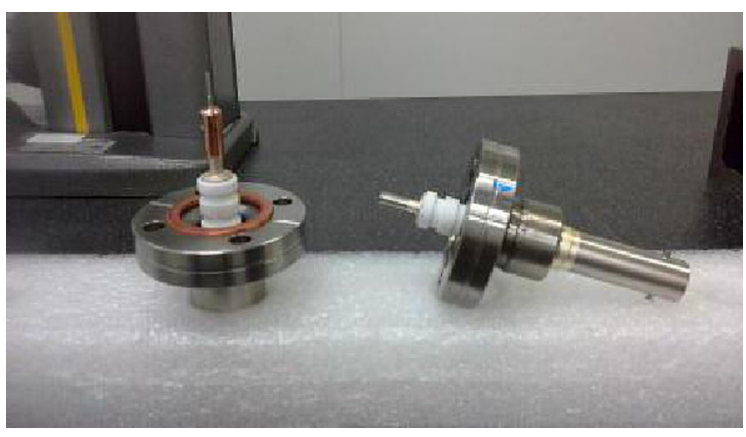

FIG. 15. Photograph of the Kyocera feedthroughs. 


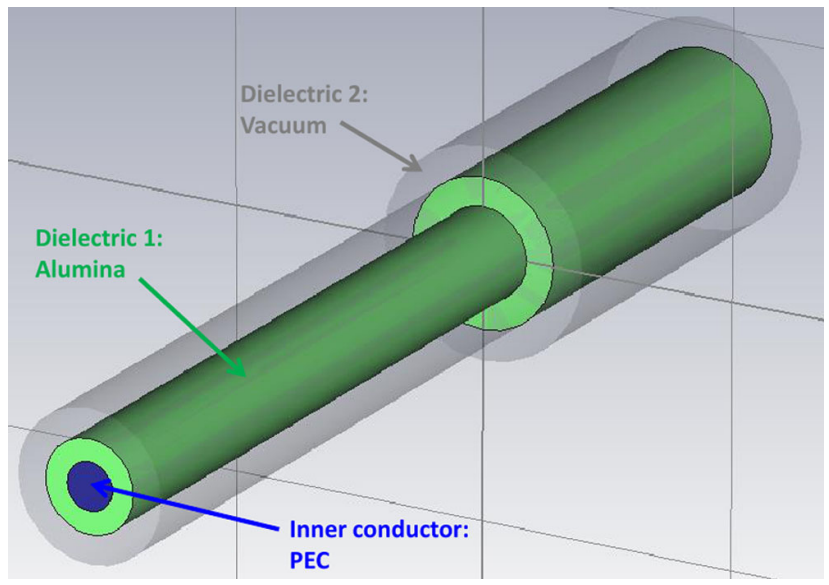

FIG. 16. Schematic of one of the Kyocera feedthroughs [20] used to connect the electrodes with the inductive adder and the loads.

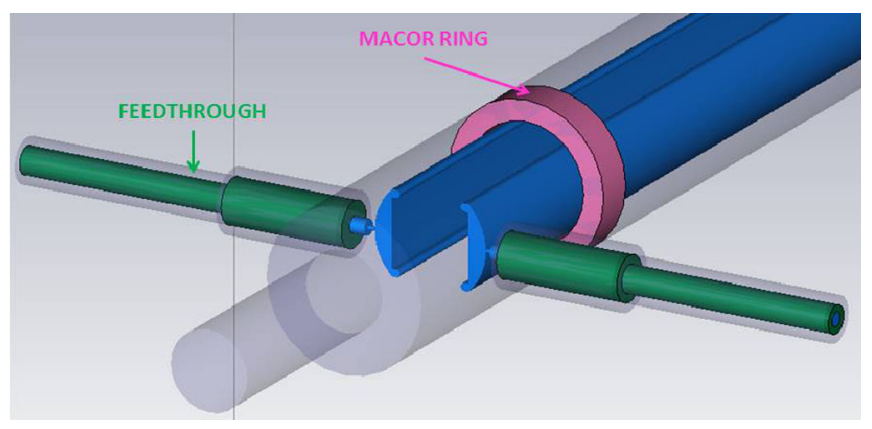

FIG. 17. CST MS model used to optimize the electrode supports and the feedthroughs for the half-moon electrodes.

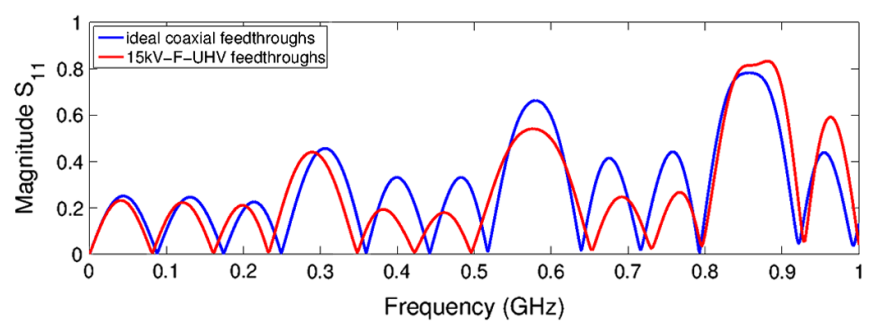

FIG. 18. Predicted magnitude of $S_{11}$ for half-moon electrode striplines with ideal coaxial feedthroughs and with Kyocera $15 \mathrm{kV}-\mathrm{F}-\mathrm{UHV}$ feedthroughs.

feedthrough and the striplines being lower than $50 \Omega$ up to $800 \mathrm{MHz}$. Furthermore, a frequency shift is observed in the maxima and minima pattern for the two models. This is due to the change in the dielectric from only vacuum for both the ideal coaxial feedthrough and the transition to the electrode, to $\mathrm{Al}_{2} \mathrm{O}_{3}$ for the Kyocera coaxial feedthrough and back to vacuum for the transition: the different dielectric constant results in a change in the velocity of the pulse signal, and therefore a frequency shift.

In addition, simulations of beam coupling impedance have been carried out: preliminary results from the model with the Kyocera feedthroughs do not show any difference compared to the model simulated using ideal $50 \Omega$ feedthroughs. Changes in the beam coupling impedance could occur at high frequencies; however, it has not been possible to run CST simulations at higher frequencies than $5 \mathrm{GHz}$.

\section{FUTURE STUDIES FOR A NEXT GENERATION PROTOTYPE OF THE STRIPLINES}

A first prototype of the CLIC DR striplines has been constructed by Trinos Vacuum Projects under the Spanish Program "Science For Industry." A picture of the striplines is shown in Fig. 19. The main goal to be achieved with this first prototype is the benchmarking of analytical approximations and simulations with measurements of the field homogeneity, power transmission, and beam coupling impedance.

\section{A. Analytical tapering studies}

If laboratory measurements indicate that beam induced electrode heating could be a problem, tapered electrodes may be required. In addition tapered striplines are expected to further improve power transmission. Furthermore, tapering will reduce the beam coupling impedance at high frequencies. Simulations of beam induced power dissipation and beam coupling impedance for tapered electrodes are presently being carried out. The results of these simulations will be compared with an analytical approximation.

Analytical equations for the longitudinal and transverse coupling impedance, $Z_{\|}$and $Z_{\perp}$, respectively, for untapered stripline beam position monitors, are shown in [17]

$$
\begin{gathered}
Z_{\|}=2 Z_{\text {even }}\left(\frac{\phi_{0}}{2 \pi}\right)^{2}\left[2 \sin ^{2}\left(\frac{\omega L}{c}\right)-i \sin \left(\frac{2 \omega L}{c}\right)\right], \\
Z_{\perp}=\left[\frac{Z_{\|}}{\omega}\right]_{\text {pair }}\left[\frac{c}{R^{2}}\right]\left[\frac{4}{\phi_{0}}\right]^{2}\left[\sin ^{2}\left(\frac{\phi_{0}}{2}\right)\right],
\end{gathered}
$$

where $\phi_{0}$ is the coverage angle of a single electrode, $\omega$ is the angular frequency, $L$ is the electrode length, and $R$ is the stripline beam pipe inner radius.

For the proposed striplines of approximately $1.6 \mathrm{~m}$ length, the even mode characteristic impedance $\left(Z_{\text {even }}\right)$ is $50 \Omega$ and the coverage angle $\phi_{0}$, for each stripline, is 2.0 and $1.8 \mathrm{rad}$, for a flat electrode and a half-moon electrode, respectively. The coverage angle can be estimated as $\phi_{0}=2 \tan ^{-1}(h / a)$.

An approximation for the beam coupling impedance of tapered electrodes is based on Eqs. (2) and (3), multiplied by an additional term [Eq. (4)], which take into account a linear taper in the coverage angle [21]:

$$
\left[\frac{\sin ^{2}\left(\frac{\omega l}{c}\right)}{\left(\frac{\omega l}{c}\right)^{2}}\right],
$$




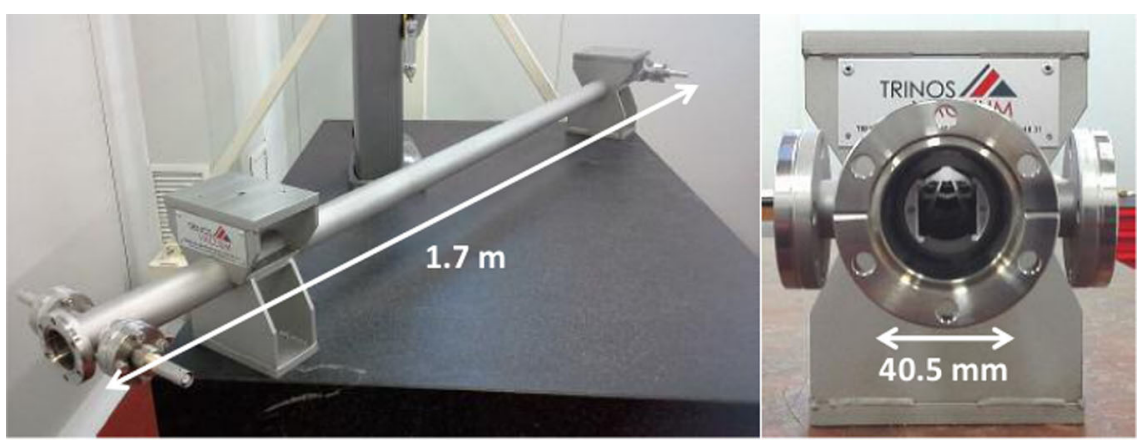

FIG. 19. Photograph of the striplines beam tube and feedthroughs (left), as well as a cross section detail showing the new half-moon electrode shape (right).

where $l$ is the taper length shown in Fig. 20. Results from the analytical equations for both untapered and tapered half-moon electrodes are shown in Fig. 21. From the analytical results, tapering the electrodes reduces the longitudinal beam coupling impedance over the whole frequency range analyzed. However, the first peak of the transverse beam coupling impedance will be higher than for untapered striplines. Simulations of beam coupling impedance with tapered electrodes will help to confirm this

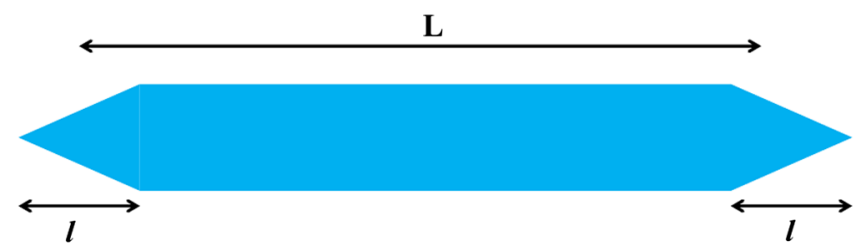

FIG. 20. Schematic of a tapered electrode, where $L$ is the electrode length to be used in Eqs. (2) and (3), and $l$ is the taper length.
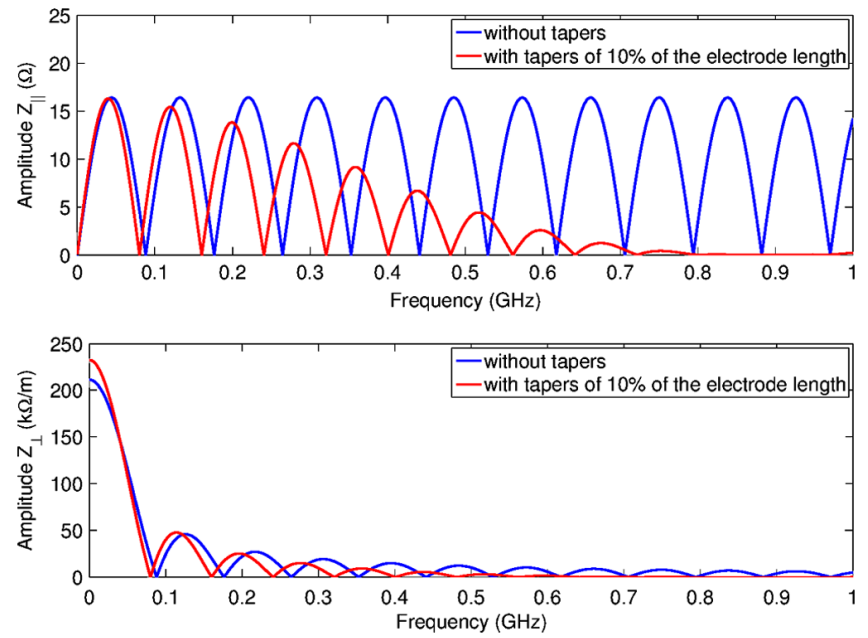

FIG. 21. Analytically calculated longitudinal beam coupling impedance (top) and transverse beam coupling impedance (bottom), for untapered and tapered half-moon electrodes with a taper length corresponding to the $10 \%$ of the electrode length. behavior. In addition, simulations of the reflection parameter and power dissipated in the case of tapered electrodes will be carried out to determine whether tapered electrodes are required for a next generation prototype.

\section{B. High frequency components}

Because of the 1 and $2 \mathrm{GHz}$ bunch spacings, and the short bunches of CLIC DRs, the beam will be affected by high frequency components up to hundreds of GHz. There may be issues of beam induced power and trapped transverse or longitudinal modes driving beam instabilities. However, up to this moment, it is very challenging to run CST simulations in this frequency range.

\section{Macor rings: Electric field}

The data sheet values for the bulk dielectric strength of Macor are $62.4 \mathrm{kV} / \mathrm{mm} \mathrm{dc}$ and $9.4 \mathrm{kV} / \mathrm{mm}$ ac [18]; however, the ac value does not include details of frequency at which the $9.4 \mathrm{kV} / \mathrm{mm}$ is valid. Tests are planned for the laboratory, in both dc and pulsed mode, to verify that the bulk dielectric strength of the Macor is sufficient. Detailed

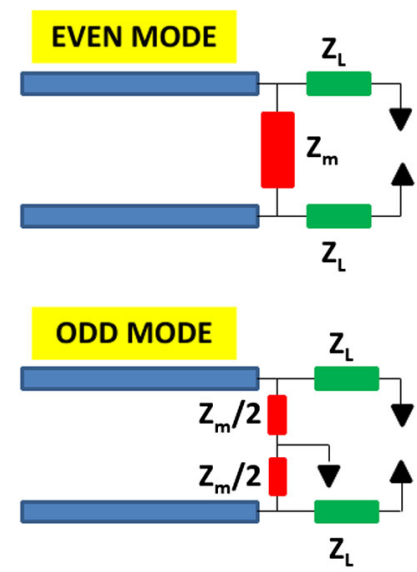

FIG. 22. Method for matching the characteristic impedance for both odd and even modes: adding a matching resistor allows for a tuning of the odd mode characteristic impedance while the even mode characteristic impedance is unchanged. 
TABLE IV. Settling time, to $\pm 0.02 \%$, as a function of pulse rise time, stripline odd mode impedance, and value of terminating resistor.

\begin{tabular}{|c|c|c|c|c|}
\hline \multirow[b]{3}{*}{ Pulse rise time } & \multicolumn{2}{|c|}{ Flat electrodes $Z_{\text {odd }}=36.8 \Omega$} & \multicolumn{2}{|c|}{ Half-moon electrodes $\mathrm{Z}_{\text {odd }}=40.9 \Omega$} \\
\hline & $\left(\mathrm{Z}_{L}\right)_{\mathrm{odd}}=50 \Omega$ & $\left(\mathrm{Z}_{L}\right)_{\mathrm{odd}}=36.8 \Omega$ & $\left(\mathrm{Z}_{L}\right)_{\mathrm{odd}}=50 \Omega$ & $\left(\mathrm{Z}_{L}\right)_{\mathrm{odd}}=40.9 \Omega$ \\
\hline & Settling time & Settling time & Settling time & Settling time \\
\hline $50 \mathrm{~ns}$ & $134 \mathrm{~ns}$ & $90 \mathrm{~ns}$ & $109 \mathrm{~ns}$ & $73 \mathrm{~ns}$ \\
\hline $100 \mathrm{~ns}$ & $113 \mathrm{~ns}$ & $77 \mathrm{~ns}$ & $78 \mathrm{~ns}$ & $66 \mathrm{~ns}$ \\
\hline $150 \mathrm{~ns}$ & $110 \mathrm{~ns}$ & $74 \mathrm{~ns}$ & $77 \mathrm{~ns}$ & $58 \mathrm{~ns}$ \\
\hline
\end{tabular}

3D simulations have been carried out to study the electric field on the surface of the Macor: the back of the electrode, head of the fixing screw, and the Macor ring have been shaped to ensure that the Macor surface field is less than $5 \mathrm{kV} / \mathrm{mm}$, for an electrode voltage of $12.5 \mathrm{kV}$. If, during laboratory testing, surface flashover occurs, surface coatings will be considered for increasing the field at which flashover occurs $[22,23]$.

\section{Nonperiodic Macor rings study}

One of the impedance mismatching sources of the striplines is the periodic discontinuities introduced by the ceramic electrode supports (Macor rings), as it has been shown in Sec. V B. Macor rings are uniformly spaced along the beam pipe and there is a significant periodic pattern caused by this uniform spacing, as shown in Fig. 12. Therefore, the possibility of using nonperiodic spacings for the electrode supports will be evaluated.

\section{E. New ideas for matching characteristic impedances}

For the optimized half-moon electrode shape, the odd mode characteristic impedance is $40.9 \Omega$. Since the inductive adder will be connected to the striplines with transmission lines of $50 \Omega$ impedance, and each stripline will be terminated with $\mathrm{Z}_{L}=50 \Omega$ (Fig. 22), there is an impedance mismatch for the odd mode at both the input and output of the electrodes: impedance mismatches can increase the settling time (Fig. 10). A new idea has been proposed to match the characteristic impedance for both the odd and even modes of excitation of the striplines, as shown in Fig. 22.

The proposal is to connect a matching resistor, $\mathrm{Z}_{m}$, between the electrodes, on the load side of the striplines. When the kicker is not pulsed the voltages induced on the electrodes are only due to the beam (even mode); the matching resistor is invisible to the even mode signals. When the kicker is pulsed, the striplines are driven with opposite polarity voltages (odd mode) and thus current flows in the matching resistor. The odd mode termination impedance is equal to

$$
\left(Z_{L}\right)_{\text {odd }}=\frac{\left(Z_{L}\right)_{\text {even }} Z_{m}}{2\left(Z_{L}\right)_{\text {even }}+Z_{m}} .
$$

This formula shows that for a terminating odd mode impedance $\left(Z_{L}\right)_{\text {odd }}=40.9 \Omega$ and a terminating even mode impedance $\left(Z_{L}\right)_{\text {even }}=Z_{L}=50 \Omega$ a matching resistor of $Z_{m}=450 \Omega$ is required.

Table IV shows settling time, to the $\pm 0.02 \%$ level, as a function of pulse rise time, odd mode impedance of the striplines $Z_{\text {odd }}$, and the value of the terminating resistor in the odd mode $\left(Z_{L}\right)_{\text {odd }}$. For the settling time simulations the following single-way delays are modeled: striplines of $7 \mathrm{~ns}$, and total for inductive adder and coaxial cables to striplines of $13 \mathrm{~ns}$. For a pulse rise time of $100 \mathrm{~ns}$, the settling time of flat electrodes terminated in $\left(\left(\mathrm{Z}_{L}\right)_{\text {odd }}=50 \Omega\right.$ is $113 \mathrm{~ns}$; the settling time is reduced to $77 \mathrm{~ns}$ by including a matching resistor so as to decrease the odd mode termination impedance to $\left(Z_{L}\right)_{\text {odd }}=36.8 \Omega$. Similarly for half-moon electrodes terminated in $\left(Z_{L}\right)_{\text {odd }}=50 \Omega$ the settling time is $78 \mathrm{~ns}$; this is reduced to $66 \mathrm{~ns}$ by including a matching resistor to decrease the odd mode termination impedance to $\left(\mathrm{Z}_{L}\right)_{\text {odd }}=40.9 \Omega$. However, a drawback of the impedance matching resistor is that it increases the pulse current which must be supplied by the inductive adder by approximately $20 \%$ for the half-moon electrodes. Even considering a reactive matching network instead of a resistive element for matching the striplines, the magnitude of the parallel combination of the $50 \Omega$ resistive termination and the parallel matching network should be $\approx 41 \Omega$ up to a frequency consistent with the content of the pulse. Thus the total current drawn from the supply may, in any case, be increased by $\approx 20 \%$ due to the matching network.

\section{CONCLUSIONS}

The electromagnetic design of the striplines, including a detailed study of odd and even mode characteristic impedances, beam coupling impedance, field homogeneity, the electrode supports, and the feedthroughs, as well as the manufacturing tolerances, has been carried out. The design studies have advanced the state of knowledge concerning stripline kickers and have thus led to a novel electrode shape. The new electrode shape provides the performance specified for the extraction kicker of the CLIC DRs: excellent field homogeneity, due to the flatness of the stripline face seen by the beam, and good power transmission, due to the reasonable matching of the 
characteristic impedance in the deflecting (odd) mode of the stripline kicker.

Tapered electrodes have been shown to permit one to obtain low beam coupling impedance and good impedance matching in the odd mode. However tapering has been demonstrated to increase the beam coupling impedance at very low frequencies, and this is undesirable for CLIC. With the novel electrode shape presented in this paper, the beam coupling impedance has been reduced at low frequencies by optimizing the nondeflecting (even) mode characteristic impedance of the stripline kicker.

A prototype of the extraction stripline kicker for the CLIC DR has been manufactured by Trinos Vacuum Projects (Valencia, Spain) and will be tested with and without beam, as well as with and without the inductive adder, in the near future. Further studies for a next prototype will include the possibility of a better matching of the characteristic impedance in the deflecting mode, as well as an analysis of tapered electrodes and nonperiodic electrode supports spacing, in order to further reduce the beam coupling impedance at high frequencies.

\section{ACKNOWLEDGMENTS}

The authors thank Trinos Vacuum-Projects S. L., responsible for building the striplines, and especially J. Gómez and D. Gutiérrez. In addition, the authors thank P. Adraktas for simulations of the electric field on the surface of the Macor. This project has been developed under CDTI (Centro para el Desarrollo Tecnológico Industrial) Contract No. IDC-20101074.

[1] CLIC Conceptual Design Report, 2012.

[2] R. Apsimon, B. Balhan, M. J. Barnes, J. Borburgh, B. Goddard, Y. Papaphilippou, and J. Uythoven, in Proceedings of the 4th International Particle Accelerator Conference, IPAC-2013, Shanghai, China, 2013 (JACoW, Shanghai, China, 2013).
[3] G. Rumolo et al., in Proceedings of the 11th European Particle Accelerator Conference, Genoa, 2008 (EPS-AG, Genoa, Italy, 2008).

[4] G. Rumolo, CERN (private communication).

[5] D. M. Pozar, Microwave Engineering, 3rd ed. (John Wiley \& Sons, New York, 2004).

[6] J. Holma, M. J. Barnes, and S. J. Ovaska, in Proceedings of the 4th International Particle Accelerator Conference, IPAC-2013, Shanghai, China, 2013 (JACoW, Shanghai, China, 2013).

[7] D. Alesini, S. Guiducci, F. Marcellini, and P. Raimondi, Phys. Rev. ST Accel. Beams 13, 111002 (2010).

[8] T. Naito, S. Araki, H. Hayano, K. Kubo, S. Kuroda, N. Terunuma, and J. Urakawa, Phys. Rev. ST Accel. Beams 14, 051002 (2011).

[9] I. Rodríguez, Ph.D. thesis, Universidad Politécnica de Madrid, 2009.

[10] T. Adachi and T. Kawakubo, Phys. Rev. ST Accel. Beams 16, 053501 (2013).

[11] A. Krasnykh, Mini Kicker Workshop in ANL, Report No. SLAC-WP-096, 2013.

[12] Ansys, www.ansys.com.

[13] CST Studio, www.cst.com.

[14] QuickField simulation code, www.quickfield.com.

[15] C. Belver-Aguilar, A. Faus-Golfe, M. J. Barnes, F. Toral, G. Rumolo, and C. Zannini, in Proceedings of the 2nd International Particle Accelerator Conference, IPAC2011, San Sebastián, Spain (EPS-AG, Spain, 2011).

[16] W. R. Blood, MECL System Design Handbook, 4th ed. (Semiconductor Component Industries, LLC (SCILLC), 2000).

[17] W. Chao and M. Tigner, Handbook of Accelerator Physics and Engineering, 3rd ed. (World Scientific, Singapore, 2006).

[18] http://www.corning.com.

[19] R. Apsimon, "CLIC RTML Collimation Systems and Beam Stabilisation”, ECFA Linear Collider Worshop 2013.

[20] http://global.kyocera.com/.

[21] S. Smith, SLAC (private communication).

[22] H. Miller, IEEE Trans. Electr. Insul. 24, 765 (1989).

[23] T. S. Sudarshan and J. D. Cross, IEEE Trans. Dielectr. Electr. Insul. EI-11, 32 (1976). 